\title{
On Initial Data in the Problem of Consistency on Cubic Lattices for $3 \times 3$ Determinants ${ }^{\star}$
}

\author{
Oleg I. MOKHOV \\ $\dagger$ Centre for Nonlinear Studies, L.D.Landau Institute for Theoretical Physics, \\ Russian Academy of Sciences, 2 Kosygina Str., Moscow, Russia \\ E-mail:mokhov@mi.ras.ru,mokhov@landau.ac.ru,mokhov@bk.ru \\ $¥$ Department of Geometry and Topology, Faculty of Mechanics and Mathematics, \\ M.V. Lomonosov Moscow State University, Moscow, Russia
}

Received January 23, 2011, in final form July 17, 2011; Published online July 26, 2011

doi:10.3842/SIGMA.2011.075

\begin{abstract}
The paper is devoted to complete proofs of theorems on consistency on cubic lattices for $3 \times 3$ determinants. The discrete nonlinear equations on $\mathbb{Z}^{2}$ defined by the condition that the determinants of all $3 \times 3$ matrices of values of the scalar field at the points of the lattice $\mathbb{Z}^{2}$ that form elementary $3 \times 3$ squares vanish are considered; some explicit concrete conditions of general position on initial data are formulated; and for arbitrary initial data satisfying these concrete conditions of general position, theorems on consistency on cubic lattices (a consistency "around a cube") for the considered discrete nonlinear equations on $\mathbb{Z}^{2}$ defined by $3 \times 3$ determinants are proved.
\end{abstract}

Key words: consistency principle; square and cubic lattices; integrable discrete equation; initial data; determinant; bent elementary square; consistency "around a cube"

2010 Mathematics Subject Classification: 39A05; 52C07; 15A15; 37K10; 11H06

\section{Introduction}

In this paper we present complete proofs of theorems on consistency on cubic lattices for $3 \times 3$ determinants. Formulations and a scheme of proofs of these theorems were given by the author in $[1,2]$, where a new, modified, consistency principle on cubic lattices for a special class of two-dimensional discrete equations defined by relations on elementary $N \times N$ squares of the square lattice $\mathbb{Z}^{2}, N>2$, was proposed. Earlier, in $[3,4,5]$, the remarkable and very natural principle of consistency on cubic lattices was proposed for discrete equations defined by relations on elementary $2 \times 2$ squares of the square lattice $\mathbb{Z}^{2}$ as an effective test singling out a certain special class of "integrable" discrete equations (see also $[6,7,8,9,10,11,12,13,14]$ ). In this paper, we consider only the discrete nonlinear equations on $\mathbb{Z}^{2}$ defined by the condition that the determinants of all $3 \times 3$ matrices of values of the corresponding scalar field $u$ at the points of the lattice $\mathbb{Z}^{2}$ that form elementary $3 \times 3$ squares vanish. We formulate some explicit concrete conditions of general position on initial data, and for arbitrary initial data satisfying these concrete conditions of general position, we prove theorems on consistency on cubic lattices (a consistency "around a cube") for the considered discrete nonlinear equations on $\mathbb{Z}^{2}$ defined by $3 \times 3$ determinants.

${ }^{\star}$ This paper is a contribution to the Proceedings of the Conference "Symmetries and Integrability of Difference Equations (SIDE-9)" (June 14-18, 2010, Varna, Bulgaria). The full collection is available at http://www.emis.de/journals/SIGMA/SIDE-9.html 


\section{Consistency principle on cubic lattices}

We consider the square lattice $\mathbb{Z}^{2}$ consisting of all points with arbitrary integer coordinates in $\mathbb{R}^{2}=\left\{\left(x_{1}, x_{2}\right) \mid x_{k} \in \mathbb{R}, k=1,2\right\}$ and complex (or real) scalar fields $u$ on the lattice $\mathbb{Z}^{2}$, $u: \mathbb{Z}^{2} \rightarrow \mathbb{C}$, defined by their values $u_{i_{1} i_{2}}, u_{i_{1} i_{2}} \in \mathbb{C}$, at each lattice point with coordinates $\left(i_{1}, i_{2}\right)$, $i_{k} \in \mathbb{Z}, k=1,2$.

We consider a class of two-dimensional discrete equations on the lattice $\mathbb{Z}^{2}$ for the field $u$ that are given by functions $Q\left(x_{1}, x_{2}, x_{3}, x_{4}\right)$ of four variables with the help of the relations

$$
Q\left(u_{i j}, u_{i+1, j}, u_{i, j+1}, u_{i+1, j+1}\right)=0, \quad i, j \in \mathbb{Z}
$$

so that in each elementary $2 \times 2$ square of the lattice $\mathbb{Z}^{2}$, i.e., in each set of lattice points with coordinates of the form $\{(i, j),(i+1, j),(i, j+1),(i+1, j+1)\}, i, j \in \mathbb{Z}$, the value of the field $u$ at one of the vertices of the square is determined by the values of the field at the other three vertices.

In this case the scalar field $u$ on the lattice $\mathbb{Z}^{2}$ is completely determined by fixing initial data, for example, on the coordinate axes of the lattice, $u_{i 0}$ and $u_{0 j}, i, j \in \mathbb{Z}$.

Here, we do not discuss conditions on the initial data $u_{i j}$ themselves that must correctly and completely determine a scalar field $u$ on the lattice $\mathbb{Z}^{2}$ for concrete discrete equations of the form (1), and also we do not discuss conditions on the functions $Q\left(x_{1}, x_{2}, x_{3}, x_{4}\right)$ for which relations (1) correctly determine a two-dimensional discrete equation on the lattice $\mathbb{Z}^{2}$ for the field $u$.

We consider the cubic lattice $\mathbb{Z}^{3}$ consisting of points with integer coordinates in $\mathbb{R}^{3}=$ $\left\{\left(x_{1}, x_{2}, x_{3}\right) \mid x_{k} \in \mathbb{R}, k=1,2,3\right\}$ and fix initial data, for example, on the coordinate axes of the lattice, $u_{i 00}, u_{0 j 0}$, and $u_{00 k}, i, j, k \in \mathbb{Z}$.

A two-dimensional discrete equation (1) is said to be consistent on the cubic lattice (see $[3,4,5,6,7])$ if for generic initial data the discrete equation (1) can be satisfied in a consistent way simultaneously on all two-dimensional coordinate sublattices of the cubic lattice $\mathbb{Z}^{3}$ that are defined by fixing one of coordinates (any of the three coordinates) of the cubic lattice. This condition is equivalent to the consistency condition on each elementary $2 \times 2 \times 2$ cube of the lattice $\mathbb{Z}^{3},\{(i+p, j+r, k+s), 0 \leq p, r, s \leq 1\}$, where $i, j$, and $k$ are arbitrary fixed integers, $i, j, k \in \mathbb{Z}$, i.e., relation (1) must be satisfied in a consistent way on all faces of any elementary $2 \times 2 \times 2$ cube of the lattice $\mathbb{Z}^{3}$ for generic initial data. In the elementary cube $\{(i, j, k), 0 \leq i, j, k \leq 1\}$ the values $u_{101}, u_{110}$, and $u_{011}$ are determined by relations (1) on the corresponding faces of the cube by the initial data $u_{000}, u_{100}, u_{010}$, and $u_{001}$, and three relations on three faces of the cube must be satisfied for the value $u_{111}$. One can consider the condition of consistency of the overdetermined system of relations for the value $u_{111}$ for generic initial data as the consistency condition for the discrete equation (1) on the cubic lattice $\mathbb{Z}^{3}$.

Here, we do not discuss all various situations in which relations (1) correctly define a twodimensional discrete equation for the field $u$ on any two-dimensional coordinate sublattice of the cubic lattice $\mathbb{Z}^{3}$; for example, one can assume for simplicity that relations (1) are invariant with respect to the full symmetry group of the square. Classifications of discrete equations of the form (1) that are consistent on the cubic lattice were studied in [6] and [10] under some additional conditions on the functions $Q\left(x_{1}, x_{2}, x_{3}, x_{4}\right)$ (see also [11]). The equation

$$
u_{i, j+1} u_{i+1, j}-u_{i+1, j+1} u_{i j}=0, \quad i, j \in \mathbb{Z}
$$

defined by the condition that the determinants of all $2 \times 2$ matrices of values of the field $u$ at the vertices of elementary $2 \times 2$ squares of the lattice $\mathbb{Z}^{2}$ vanish, is an example of such a twodimensional nonlinear discrete equation that is consistent on the cubic lattice. Equation (2) is linear with respect to each variable and invariant with respect to the full symmetry group of 
the square. Fixing arbitrary nonzero initial data $u_{i 0}$ and $u_{0 j}, i, j \in \mathbb{Z}$, on the coordinate axes of the lattice $\mathbb{Z}^{2}$ completely determines a field $u$ on the lattice $\mathbb{Z}^{2}$ satisfying the discrete nonlinear equation (2), and fixing arbitrary nonzero initial data $u_{i 00}, u_{0 j 0}$, and $u_{00 k}, i, j, k \in \mathbb{Z}$, on the coordinate axes of the lattice $\mathbb{Z}^{3}$ completely determines a field $u$ on the lattice $\mathbb{Z}^{3}$ satisfying the discrete nonlinear equation (2) on all two-dimensional coordinate sublattices of the cubic lattice $\mathbb{Z}^{3}$; i.e., relations (2) are satisfied in a consistent way on all faces of each elementary $2 \times 2 \times 2$ cube of the lattice $\mathbb{Z}^{3}$ for arbitrary nonzero initial data. The integrability (in the broadest sense of the word) of the discrete nonlinear equation (2) is obvious, since it can be easily linearized: $\ln u_{i, j+1}+\ln u_{i+1, j}-\ln u_{i+1, j+1}-\ln u_{i j}=0, i, j \in \mathbb{Z}$. Discrete nonlinear equations defined by determinants of higher orders are also $\mathcal{C}$-integrable and it is not a problem to write their general solution for generic initial data, but in any case they are much more complicated, nonlinearizable, and the problem on their consistency on cubic lattice is very nontrivial (see $[1,2])$.

\section{Relations on elementary $3 \times 3$ squares of the lattice $\mathbb{Z}^{2}$ and consistency conditions}

We will use the following definition everywhere in this paper. An elementary $N \times N$ square of the square lattice $\mathbb{Z}^{2}$ is a set of points of the lattice $\mathbb{Z}^{2}$ with coordinates $\{(i+s, j+r), 0 \leq s, r \leq N-1\}$, where $i, j$ is an arbitrary fixed pair of integers, $i, j \in \mathbb{Z}, N \geq 2$.

Let us consider a discrete equation on $\mathbb{Z}^{2}$ defined by a relation for the values of the field $u$ at the points of the lattice $\mathbb{Z}^{2}$ that form elementary $3 \times 3$ squares:

$$
Q\left(u_{i j}, \ldots, u_{i+s, j+r}, \ldots, u_{i+2, j+2}\right)=0, \quad 0 \leq s, r \leq 2, \quad i, j \in \mathbb{Z},
$$

so that in each elementary $3 \times 3$ square of the lattice $\mathbb{Z}^{2}$, i.e., in each set of lattice points with coordinates of the form $\{(i, j),(i+1, j),(i+2, j),(i, j+1),(i+1, j+1),(i+2, j+1),(i, j+2)$, $(i+1, j+2),(i+2, j+2)\}, i, j \in \mathbb{Z}$, the value of the field $u$ at one of the points of this elementary $3 \times 3$ square is determined by the values of the field at the other eight points.

For definiteness, one can require, for example, that relations (3) are invariant with respect to the full symmetry group of the configuration of points of the lattice $\mathbb{Z}^{2}$ that form elementary $3 \times 3$ squares (obviously, this group of symmetries coincides with the full symmetry group of the usual square). For any discrete equation of the form (3), fixing generic initial data, for example, on two bands along the coordinate axes of the lattice $\mathbb{Z}^{2},\{(i, 0),(i, 1), i \in \mathbb{Z}\}$ and $\{(0, j),(1, j), j \in \mathbb{Z}\}$, completely determines a field $u$ on $\mathbb{Z}^{2}$ that satisfies this equation.

Quite similarly, discrete equations on $\mathbb{Z}^{2}$ given by relations for the values of the field $u$ at the points of the lattice $\mathbb{Z}^{2}$ that form elementary $N \times N$ squares can be defined for an arbitrary $N \geq 2$. For definiteness, one can again require, for example, that the relations are invariant with respect to the full symmetry group of the configuration of points of the lattice $\mathbb{Z}^{2}$ that form elementary $N \times N$ squares (moreover, it is also obvious that for any $N \geq 2$ the full symmetry group of the configuration of points of the lattice $\mathbb{Z}^{2}$ that form elementary $N \times N$ squares coincides with the full symmetry group of the usual square).

We consider the cubic lattice $\mathbb{Z}^{3}$ and the consistency condition for discrete equations of the form (3) on all two-dimensional coordinate sublattices of the cubic lattice $\mathbb{Z}^{3}$. Initial data can be specified, for example, at the following lattice points that are situated on 12 straight lines going along the coordinate axes of the lattice $\mathbb{Z}^{3}:(i, 0,0),(i, 1,0),(i, 0,1),(i, 1,1),(0, j, 0),(1, j, 0)$, $(0, j, 1),(1, j, 1),(0,0, k),(1,0, k),(0,1, k)$, and $(1,1, k), i, j, k \in \mathbb{Z}$. The values of the field $u$ at all other points of the cubic lattice $\mathbb{Z}^{3}$ must then be correctly determined in a consistent way by relations (3) on all elementary $3 \times 3$ squares of all two-dimensional coordinate sublattices of the cubic lattice $\mathbb{Z}^{3}$ for generic initial data. In the elementary cube $\{(i, j, k), 0 \leq i, j, k \leq$ $2\}$ the values $u_{202}, u_{212}, u_{220}, u_{221}, u_{022}$, and $u_{122}$ are determined using relations $(3)$ on the 
corresponding elementary $3 \times 3$ squares situated in the cube under consideration (three faces of the cube that are situated on the coordinate planes and three middle normal sections of the cube) by the initial data $u_{000}, u_{100}, u_{200}, u_{010}, u_{110}, u_{210}, u_{001}, u_{101}, u_{201}, u_{011}, u_{111}, u_{211}, u_{020}$, $u_{120}, u_{021}, u_{121}, u_{002}, u_{102}, u_{012}$, and $u_{112}$, and three relations must hold simultaneously on three other faces of the cube for the value $u_{222}$. One can assume that the consistency condition on the cubic lattice $\mathbb{Z}^{3}$ for any discrete equation of the form (3) is the consistency condition of the corresponding overdetermined system of relations on the value $u_{222}$ for generic initial data.

Quite similarly, for an arbitrary $N \geq 2$, one can define the consistency condition on the cubic lattice $\mathbb{Z}^{3}$ for discrete equations on $\mathbb{Z}^{2}$ given by relations on the values of the field $u$ at the points of the lattice $\mathbb{Z}^{2}$ that form elementary $N \times N$ squares. The values of the field $u$ at all points of the cubic lattice $\mathbb{Z}^{3}$ must be correctly determined in a consistent way by relations on all elementary $N \times N$ squares of all two-dimensional coordinate sublattices of the cubic lattice $\mathbb{Z}^{3}$ for generic initial data. The consistency of discrete equations on the lattice $\mathbb{Z}^{2}$ that are given by relations on the values of the field $u$ at the points of the lattice $\mathbb{Z}^{2}$ that form elementary $N \times N$ squares can be considered on one elementary $N \times N \times N$ cube (the consistency of the relations on all faces and all normal sections of the cube that are parallel to the coordinate planes for generic initial data specified in the cube).

Let us consider the discrete nonlinear equation on $\mathbb{Z}^{2}$ defined by the condition that the determinants of all $3 \times 3$ matrices of values of the field $u$ at the points of the lattice $\mathbb{Z}^{2}$ that form elementary $3 \times 3$ squares vanish:

$$
\begin{aligned}
& u_{i, j+2} u_{i+1, j+1} u_{i+2, j}+u_{i, j+1} u_{i+1, j} u_{i+2, j+2}+u_{i, j} u_{i+1, j+2} u_{i+2, j+1} \\
& -u_{i, j} u_{i+1, j+1} u_{i+2, j+2}-u_{i, j+2} u_{i+1, j} u_{i+2, j+1}-u_{i, j+1} u_{i+1, j+2} u_{i+2, j}=0, \quad i, j \in \mathbb{Z} .
\end{aligned}
$$

Equation (4) is linear with respect to each variable and invariant with respect to the full symmetry group of the configuration of points of the lattice $\mathbb{Z}^{2}$ that form elementary $3 \times 3$ squares.

It is not difficult to check that for generic initial data the above-considered consistency condition on the cubic lattice is not satisfied for the discrete equation (4), and, in this sense, the discrete nonlinear equation (4) is not consistent on two-dimensional coordinate sublattices of the cubic lattice $\mathbb{Z}^{3}$.

We note that for such setting of the consistency problem on the cubic lattice for discrete equations of the form (3) we have the following: in the elementary $3 \times 3 \times 3$ cube $\{(i, j, k), 0 \leq$ $i, j, k \leq 2\}$ of the lattice $\mathbb{Z}^{3}$, given initial data of values of the field $u$ at 20 points of this elementary $3 \times 3 \times 3$ cube, one can determine the values of the field $u$ at the other seven points of this elementary $3 \times 3 \times 3$ cube by relations (3) (nine relations on six faces and on three middle normal sections of the cube), and only for the value of the field at one of the points one obtains an overdetermined system consisting of three relations on three distinct elementary $3 \times 3$ squares.

\section{$4 \quad$ Bent elementary $3 \times 3$ squares and modified consistency conditions}

We consider a discrete equation of the form (3) and require that the discrete equation is satisfied not only on all two-dimensional coordinate sublattices of the cubic lattice $\mathbb{Z}^{3}$, but also on all unions of two arbitrary intersecting two-dimensional coordinate sublattices of the cubic lattice $\mathbb{Z}^{3}$; i.e., the corresponding elementary $3 \times 3$ squares on which the discrete equation of the form (3) is considered can be bent at a right angle along any of two middle lines of the elementary $3 \times 3$ square passing from one two-dimensional coordinate sublattice to another so that all points of bent elementary $3 \times 3$ squares are situated at lattice points (one of lines of any bent elementary

$3 \times 3$ square is situated on one of two intersecting two-dimensional coordinate sublattices of the cubic lattice $\mathbb{Z}^{3}$, one of the lines is situated on the second of these two sublattices, and the 
middle line which this elementary square is bent along is situated on the intersection of these two intersecting two-dimensional coordinate sublattices), for example, $\{(i, 0,0),(i, 1,0),(i, 0,1), i=$ $0,1,2\},\{(0, j, 0),(1, j, 0),(0, j, 1), j=0,1,2\}$, and $\{(0,0, k),(1,0, k),(0,1, k), k=0,1,2\}$ (bent elementary $3 \times 3$ squares). We will consider relation (3) on all elementary $3 \times 3$ squares (bent and unbent) all points of which are situated at lattice points. In this case initial data can be specified, for example, at the following points of the cubic lattice $\mathbb{Z}^{3}:(i, 0,0),(i, 0,1),(0, j, 0)$, $(0, j, 1),(0,0, k),(1,0, k),(1,1,0)$, and $(1,1,1), i, j, k \in \mathbb{Z}$. The values of the field $u$ at all other points of the cubic lattice $\mathbb{Z}^{3}$ must then be correctly determined in a consistent way by relations (3) on all elementary $3 \times 3$ squares (including all bent elementary $3 \times 3$ squares) of all unions of two arbitrary intersecting two-dimensional coordinate sublattices of the cubic lattice $\mathbb{Z}^{3}$ for generic initial data. In the elementary cube $\{(i, j, k), 0 \leq i, j, k \leq 2\}$ the values $u_{012}, u_{022}, u_{112}, u_{202}, u_{12 k}, u_{21 k}$, and $u_{22 k}, 0 \leq k \leq 2$, are determined by the initial data $u_{000}$, $u_{100}, u_{200}, u_{001}, u_{110}, u_{010}, u_{101}, u_{201}, u_{111}, u_{011}, u_{002}, u_{020}, u_{021}$, and $u_{102}$ and by overdetermined systems generated by relations (3) on elementary $3 \times 3$ squares (including all bent elementary $3 \times 3$ squares) that are situated in the cube under consideration (six faces, three middle normal sections of the cube, and 48 bent elementary $3 \times 3$ squares). There are 48 distinct bent elementary $3 \times 3$ squares in any elementary $3 \times 3 \times 3$ cube, which can be easily counted by the bending edges of the bent elementary $3 \times 3$ squares: to each of the 12 edges of the cube, there corresponds one bent elementary $3 \times 3$ square in the cube; to each of the 12 middle lines of points on the faces of the cube $(2 \times 6)$, there correspond two distinct bent elementary $3 \times 3$ squares in the cube; and to each of the three interior lines of points in the cube that connect the centres of opposite faces of the cube, there correspond four distinct bent elementary $3 \times 3$ squares in the cube. One can assume that the consistency condition on the cubic lattice $\mathbb{Z}^{3}$ for any discrete equation of the form (3) (the global consistency) is the consistency condition of the corresponding overdetermined system of relations on the values of the field $u$ in the elementary cube $\{(i, j, k), 0 \leq i, j, k \leq 2\}$ for generic initial data (the local consistency). The corresponding discrete equations will also be called consistent on the cubic lattice.

We note that for this new setting of the consistency problem on the cubic lattice for discrete equations of the form (3) we have the following: in the elementary $3 \times 3 \times 3$ cube $\{(i, j, k), 0 \leq$ $i, j, k \leq 2\}$ of the lattice $\mathbb{Z}^{3}$, given initial data of values of the field $u$ at 14 points of this elementary $3 \times 3 \times 3$ cube, one can determine the values of the field $u$ at the other 13 points of this elementary $3 \times 3 \times 3$ cube by relations (3) ( 57 relations on six faces, on three middle normal sections of the cube, and on 48 bent elementary $3 \times 3$ squares), which constitute in this case a highly overdetermined system of relations.

We also note that the global consistency follows from the local consistency, i.e., if the consistency condition is fulfilled for generic initial data in the elementary cube $\{(i, j, k), 0 \leq i, j, k \leq 2\}$, then it is fulfilled for generic initial data everywhere in the cubic lattice $\mathbb{Z}^{3}$, i.e., in each elementary $3 \times 3 \times 3$ cube $\left\{\left(i_{0}+i, j_{0}+j, k_{0}+k\right), 0 \leq i, j, k \leq 2\right\}, i_{0}, j_{0}, k_{0} \in \mathbb{Z}$. We give here a formal scheme of proof. The number of given initial data in an arbitrary elementary $3 \times 3 \times 3$ cube $\left\{\left(i_{0}+i, j_{0}+j, k_{0}+k\right), 0 \leq i, j, k \leq 2\right\}, i_{0}, j_{0}, k_{0} \in \mathbb{Z}$, is not more than 14 as we have in the "main" elementary cube $\{(i, j, k), 0 \leq i, j, k \leq 2\}$. We start from the "main" elementary cube $\{(i, j, k), 0 \leq i, j, k \leq 2\}$, where the consistency condition is fulfilled for generic initial data as the local consistency by our assumption, and then we will consider some special shifts of elementary $3 \times 3 \times 3$ cubes step by step from the "main" elementary cube $\{(i, j, k), 0 \leq i, j, k \leq 2\}$ until fill all the cubic lattice $\mathbb{Z}^{3}$. There are three essentially different situations, when after shifting we obtain new 9 points of the cubic lattice $\mathbb{Z}^{3}$ (the face of the shifted elementary $3 \times 3 \times 3$ cube), new 3 points (the edge of the shifted elementary $3 \times 3 \times 3$ cube) or new only one point (the vertice of the shifted elementary $3 \times 3 \times 3$ cube). First of all, we consider 6 shifted elementary $3 \times 3 \times 3$ cubes ( 6 elementary $3 \times 3 \times 3$ cubes shifted to the side of each of the faces of the "main" elementary cube $\{(i, j, k), 0 \leq i, j, k \leq 2\})$. In these elementary $3 \times 3 \times 3$ cubes we 
have some given initial data (not more than 14) and some values of the field $u$ determined in the "main" elementary cube $\{(i, j, k), 0 \leq i, j, k \leq 2\}$. Let us consider any of these elementary $3 \times 3 \times 3$ cubes and prove that the consistency condition is also fulfilled in it. We consider some determined values as new initial data for this elementary $3 \times 3 \times 3$ cube, first of all, we take given initial data and if it is necessary (i.e., if the number of given initial data in this elementary $3 \times 3 \times 3$ cube is less than 14 ), add to given initial data the corresponding values determined in the "main" elementary cube $\{(i, j, k), 0 \leq i, j, k \leq 2\}$. It is simple to check that we always can do this. Then we can determine all values in this elementary $3 \times 3 \times 3$ cube in a consistent way by our assumption of the local consistency. After that we must prove that the values determined simultaneously from the "main" elementary cube $\{(i, j, k), 0 \leq i, j, k \leq 2\}$ and from the elementary $3 \times 3 \times 3$ cube under consideration coincide. It follows from the fact that all these values can be determined step by step from elementary $3 \times 3$ squares (bent and unbent) situated in both these elementary $3 \times 3 \times 3$ cubes simultaneously. Similarly, we prove step by step that the consistency condition is fulfilled in any elementary $3 \times 3 \times 3$ cube obtained from the "main" elementary cube $\{(i, j, k), 0 \leq i, j, k \leq 2\}$ by a shift along the coordinate axes (along the given bands of initial data). Then we prove step by step that the consistency condition is fulfilled in any elementary $3 \times 3 \times 3$ cube obtained from the considered elementary $3 \times 3 \times 3$ cubes by shifts in the corresponding coordinate planes. This is the second type of our shifts. Again we must consider some initial data in each elementary $3 \times 3 \times 3$ cube and determine all values in it in a consistent way by our assumption of the local consistency. All the values determined simultaneously from different elementary $3 \times 3 \times 3$ cubes coincide since all these values can be determined step by step from elementary $3 \times 3$ squares (bent and unbent) situated in both these elementary $3 \times 3 \times 3$ cubes simultaneously. Similarly, we prove step by step that the consistency condition is fulfilled for the shifts of the third type filling all the cubic lattice $\mathbb{Z}^{3}$.

Quite similarly, for an arbitrary $N>2$, one can define the corresponding modified consistency condition on the cubic lattice $\mathbb{Z}^{3}$ for discrete equations on the square lattice $\mathbb{Z}^{2}$ that are given by relations on the values of the field $u$ at the points of the lattice $\mathbb{Z}^{2}$ that form elementary $N \times N$ squares (including any bent elementary $N \times N$ squares). Moreover, for $N>3$, one can, generally speaking, allow a larger number (up to $N-2$ ) of bendings of elementary $N \times N$ squares in the cubic lattice $\mathbb{Z}^{3}$ (in this case each elementary $N \times N$ square can be bent in the cubic lattice simultaneously along up to $N-2$ parallel lines of the same type, each bending being to one of the two possible different sides).

\section{Consistency on cubic lattices for $3 \times 3$ determinants}

The following basic theorem holds.

Theorem 1 ([1]). For arbitrary generic initial data, the nonlinear discrete equation (4) can be satisfied in a consistent way on all unions of pairs of arbitrary intersecting two-dimensional coordinate sublattices of the cubic lattice $\mathbb{Z}^{3}$; i.e., the discrete nonlinear equation (4) is consistent on the cubic lattice $\mathbb{Z}^{3}$.

Proof. Here we give the strict proof of consistency "around the elementary cube" (the local consistency) for generic initial data. Let us consider the elementary cube $\{(i, j, k), 0 \leq i, j, k \leq 2\}$ of the cubic lattice $\mathbb{Z}^{3}$ and specify generic initial data at the following points of this elementary cube: $(i, 0,0),(i, 0,1),(0, j, 0),(0, j, 1),(0,0, k),(1,0, k),(1,1,0)$, and $(1,1,1), 0 \leq i, j, k \leq 2$. In particular, it is sufficient to require that the following condition on initial data is fulfilled: in all elementary $3 \times 3$ squares (bent and unbent) situated in the elementary cube under consideration, all $2 \times 2$ minors that are completely formed by only initial data are not equal to zero (this is a condition of general position for initial data). In this paper, we will assume that precisely this concrete condition of general position on initial data is fulfilled. We note that the condition 
on initial data, when no four values $u_{a}, u_{b}, u_{c}, u_{d}$ of initial data from distinct points $a, b, c$, $d$ of the cube under consideration satisfy the relation $u_{a} u_{b}=u_{c} u_{d}$ (i.e., if we arrange values of initial data from distinct points of the cube under consideration in all points of elementary $2 \times 2$ square in an arbitrary way, then all the determinants of the corresponding $2 \times 2$ matrices obtained by this procedure are not equal to zero), is also a condition of general position for initial data. We require the fulfillment of a weaker condition on initial data, when only some of these determinants, namely, only those from them that are $2 \times 2$ minors formed by initial data in the $3 \times 3$ matrices of values of the field at the points of the elementary $3 \times 3$ squares (bent and unbent) situated in the elementary cube under consideration, must not be equal to zero. We will distinguish the following three different types of lines of lattice points in the elementary cube under consideration: the lines parallel to the $x$-axis, i.e., the sets of points of the form $\{(i, r, s), 0 \leq i \leq 2\}$, where $(r, s)$ are fixed ordered pairs of integers, $0 \leq r, s \leq 2$, that number the lines in this elementary cube that are parallel to the $x$-axis (the $x$-type lines); the lines parallel to the $y$-axis, i.e., the sets of points of the form $\{(r, j, s), 0 \leq j \leq 2\}$, where $(r, s)$ are fixed ordered pairs of integers, $0 \leq r, s \leq 2$, that number the lines in the elementary cube under consideration that are parallel to the $y$-axis (the $y$-type lines); and the lines parallel to the $z$-axis, i.e., the sets of points of the form $\{(r, s, k), 0 \leq k \leq 2\}$, where $(r, s)$ are fixed ordered pairs of integers, $0 \leq r, s \leq 2$, that number the lines in the elementary cube under consideration that are parallel to the $z$-axis (the $z$-type lines). The specified initial data fill a pair of lines of each of these three types (a pair of lines parallel to the corresponding coordinate axis for each of the coordinate axes). We will consider all these lines as basic ones: $\{(i, 0,0), 0 \leq i \leq 2\}$ and $\{(i, 0,1), 0 \leq i \leq 2\}$ (the basic $x$-type lines); $\{(0, j, 0), 0 \leq j \leq 2\}$ and $\{(0, j, 1), 0 \leq j \leq 2\}$ (the basic $y$-type lines); and $\{(0,0, k), 0 \leq k \leq 2\}$ and $\{(1,0, k), 0 \leq k \leq 2\}$ (the basic $z$-type lines). The vectors of values of the field $u$ at the points of the basic lines will be called basic vectors (of the corresponding type). Note that the vectors of values of the field $u$ at the points of the basic lines of each type are linearly independent, since otherwise the corresponding $2 \times 2$ minors formed by initial data must be equal to zero. Therefore, basic vectors of each type are linearly independent. Given arbitrary generic initial data, we will determine the values of the scalar field $u$ at the remaining points of the elementary cube under consideration according to relations (4) and mark the points at which the values of the field have already been found. We will also shade each line in this elementary cube if the vector of values of the field $u$ at the points of this line is a linear combination of the vectors of values of the field $u$ at the points of the two basic lines of the same type (for the coordinates of vectors of values of the field $u$ at the points of lines of the same type, there is a natural ascending order of the respective coordinate $x, y$ or $z$ ). First of all, in the elementary cube under consideration we must mark all lattice points at which the initial data are given and shade all basic lines of all three types by the very definition of this procedure. It is obvious that if carrying out such a procedure for generic initial data yields all the lattice points marked and all the lines of all the types shaded in the elementary cube under consideration, then the theorem will be proved, because in this case, for any three lines of the same type in this elementary cube (and, hence, for any elementary $3 \times 3$ square in this elementary cube, bent or unbent), the determinant of the matrix of values of the scalar field $u$ at the points of these lines will vanish, and this is even more than is required for the consistency of the corresponding discrete equation. Thus, in this case, as a matter of fact, we will prove even a considerably stronger principle of consistency on the cubic lattice $\mathbb{Z}^{3}$ for determinants and for the nonlinear discrete equation (4). It remains to shade all the lines of all the types in the elementary cube under consideration. For this purpose, it is necessary to consider consecutively at least 13 elementary $3 \times 3$ squares (bent and unbent) of our elementary cube determining values of the field $u$ at 13 unmarked points.

Let us consider the elementary $3 \times 3$ square $\{(i, 0,0),(i, 0,1),(i, 0,2), i=0,1,2\}$ in our cube (a face of the cube). In this elementary square the values of the field $u$ are given at eight points and the value of the field $u$ at the remaining ninth point $(2,0,2)$ is determined by relation (4), 
i.e., by the condition that the determinant of the matrix of values of the field at the lattice points of this elementary $3 \times 3$ square vanishes, because the corresponding $2 \times 2$ minor formed by initial data is not equal to zero. Since basic vectors of the same type are linearly independent, the vector of values of the field $u$ at the points of the line $\{(i, 0,2), 0 \leq i \leq 2\}$ is a linear combination of the vectors of values of the field $u$ at the points of the two basic lines of the same type, $\{(i, 0,0), 0 \leq i \leq 2\}$ and $\{(i, 0,1), 0 \leq i \leq 2\}$, situated in the given elementary $3 \times 3$ square; i.e., we can mark the point $(2,0,2)$ and shade the line $\{(i, 0,2), 0 \leq i \leq 2\}$. Moreover, the obtained vector of values of the field $u$ at the points of the line $\{(i, 0,2), 0 \leq i \leq 2\}$ forms a linearly independent pair of vectors with each of the basic vectors of the same type, since otherwise the corresponding $2 \times 2$ minors formed by initial data must be equal to zero.

Similarly, since basic vectors of the same type are linearly independent, it follows immediately from vanishing the determinant of the matrix of values of the field at the lattice points of this elementary $3 \times 3$ square that the vector of values of the field $u$ at the points of the line $\{(2,0, k), 0 \leq k \leq 2\}$ is a linear combination of the vectors of values of the field $u$ at the points of the other two lines of this elementary $3 \times 3$ square, namely, the two basic lines of the same type, $\{(0,0, k), 0 \leq k \leq 2\}$ and $\{(1,0, k), 0 \leq k \leq 2\}$, situated in the given elementary $3 \times 3$ square; i.e., we can shade the line $\{(2,0, k), 0 \leq k \leq 2\}$. Moreover, the obtained vector of values of the field $u$ at the points of the line $\{(2,0, k), 0 \leq k \leq 2\}$ forms a linearly independent pair of vectors with each of the basic vectors of the same type, since otherwise the corresponding $2 \times 2$ minors formed by initial data must be equal to zero.

Let us consider the bent elementary $3 \times 3$ square $\{(1,0, k),(0,0, k),(0,1, k), k=0,1,2\}$ in our cube. In this elementary square the values of the field $u$ are given at eight points and the value of the field $u$ at the remaining ninth point $(0,1,2)$ is determined by relation (4), i.e., by the condition that the determinant of the matrix of values of the field at the lattice points of this bent elementary $3 \times 3$ square vanishes, because the corresponding $2 \times 2$ minor formed by initial data is not equal to zero. Since basic vectors of the same type are linearly independent, the vector of values of the field $u$ at the points of the line $\{(0,1, k), 0 \leq k \leq 2\}$ is a linear combination of the vectors of values of the field $u$ at the points of the two basic lines of the same type, $\{(0,0, k), 0 \leq k \leq 2\}$ and $\{(1,0, k), 0 \leq k \leq 2\}$, situated in the given bent elementary $3 \times 3$ square; i.e., we can mark the point $(0,1,2)$ and shade the line $\{(0,1, k), 0 \leq k \leq 2\}$. Moreover, the obtained vector of values of the field $u$ at the points of the line $\{(0,1, k), 0 \leq k \leq 2\}$ forms a linearly independent pair of vectors with each of the basic vectors of the same type, since otherwise the corresponding $2 \times 2$ minors formed by initial data must be equal to zero.

Now we consider another bent elementary $3 \times 3$ square $\{(1,1, k),(1,0, k),(0,0, k), k=0,1,2\}$ in our cube. In this elementary square the values of the field $u$ are given at eight points and the value of the field $u$ at the remaining ninth point $(1,1,2)$ is determined by relation (4), i.e., by the condition that the determinant of the matrix of values of the field at the lattice points of this bent elementary $3 \times 3$ square vanishes, because the corresponding $2 \times 2$ minor formed by initial data is not equal to zero. Since basic vectors of the same type are linearly independent, the vector of values of the field $u$ at the points of the line $\{(1,1, k), 0 \leq k \leq 2\}$ is a linear combination of the vectors of values of the field $u$ at the points of the two basic lines of the same type, $\{(1,0, k), 0 \leq k \leq 2\}$ and $\{(0,0, k), 0 \leq k \leq 2\}$, situated in the given bent elementary $3 \times 3$ square; i.e., we can mark the point $(1,1,2)$ and shade the line $\{(1,1, k), 0 \leq k \leq 2\}$. Moreover, the obtained vector of values of the field $u$ at the points of the line $\{(1,1, k), 0 \leq k \leq 2\}$ forms a linearly independent pair of vectors with each of the basic vectors of the same type, since otherwise the corresponding $2 \times 2$ minors formed by initial data must be equal to zero.

Let us consider one more bent elementary $3 \times 3$ square $\{(i, 0,0),(i, 0,1),(i, 1,1), i=0,1,2\}$ in our cube. In this elementary square the values of the field $u$ are given at eight points and the value of the field $u$ at the remaining ninth point $(2,1,1)$ is determined by relation (4), i.e., by the condition that the determinant of the matrix of values of the field at the lattice points 
of this bent elementary $3 \times 3$ square vanishes, because the corresponding $2 \times 2$ minor formed by initial data is not equal to zero. Since basic vectors of the same type are linearly independent, the vector of values of the field $u$ at the points of the line $\{(i, 1,1), 0 \leq i \leq 2\}$ is a linear combination of the vectors of values of the field $u$ at the points of the two basic lines of the same type, $\{(i, 0,0), 0 \leq i \leq 2\}$ and $\{(i, 0,1), 0 \leq i \leq 2\}$, situated in the given bent elementary $3 \times 3$ square; i.e., we can mark the point $(2,1,1)$ and shade the line $\{(i, 1,1), 0 \leq i \leq 2\}$. Moreover, the obtained vector of values of the field $u$ at the points of the line $\{(i, 1,1), 0 \leq i \leq 2\}$ forms a linearly independent pair of vectors with each of the basic vectors of the same type, since otherwise the corresponding $2 \times 2$ minors formed by initial data must be equal to zero.

Let us consider the next bent elementary $3 \times 3$ square $\{(i, 0,1),(i, 0,0),(i, 1,0), i=0,1,2\}$ in our cube. In this elementary square the values of the field $u$ are given at eight points and the value of the field $u$ at the remaining ninth point $(2,1,0)$ is determined by relation (4), i.e., by the condition that the determinant of the matrix of values of the field at the lattice points of this bent elementary $3 \times 3$ square vanishes, because the corresponding $2 \times 2$ minor formed by initial data is not equal to zero. Since basic vectors of the same type are linearly independent, the vector of values of the field $u$ at the points of the line $\{(i, 1,0), 0 \leq i \leq 2\}$ is a linear combination of the vectors of values of the field $u$ at the points of the two basic lines of the same type, $\{(i, 0,0), 0 \leq i \leq 2\}$ and $\{(i, 0,1), 0 \leq i \leq 2\}$, situated in the given bent elementary $3 \times 3$ square; i.e., we can mark the point $(2,1,0)$ and shade the line $\{(i, 1,0), 0 \leq i \leq 2\}$. Moreover, the obtained vector of values of the field $u$ at the points of the line $\{(i, 1,0), 0 \leq i \leq 2\}$ forms a linearly independent pair of vectors with each of the basic vectors of the same type, since otherwise the corresponding $2 \times 2$ minors formed by initial data must be equal to zero.

Let us consider one more bent elementary $3 \times 3$ square $\{(1, j, 0),(0, j, 0),(0, j, 1), j=0,1,2\}$ in our cube. In this elementary square the values of the field $u$ are given at eight points and the value of the field $u$ at the remaining ninth point $(1,2,0)$ is determined by relation (4), i.e., by the condition that the determinant of the matrix of values of the field at the lattice points of this bent elementary $3 \times 3$ square vanishes, because the corresponding $2 \times 2$ minor formed by initial data is not equal to zero. Since basic vectors of the same type are linearly independent, the vector of values of the field $u$ at the points of the line $\{(1, j, 0), 0 \leq j \leq 2\}$ is a linear combination of the vectors of values of the field $u$ at the points of the two basic lines of the same type, $\{(0, j, 0), 0 \leq j \leq 2\}$ and $\{(0, j, 1), 0 \leq j \leq 2\}$, situated in the given bent elementary $3 \times 3$ square; i.e., we can mark the point $(1,2,0)$ and shade the line $\{(1, j, 0), 0 \leq j \leq 2\}$. Moreover, the obtained vector of values of the field $u$ at the points of the line $\{(1, j, 0), 0 \leq j \leq 2\}$ forms a linearly independent pair of vectors with each of the basic vectors of the same type, since otherwise the corresponding $2 \times 2$ minors formed by initial data must be equal to zero.

Let us consider the next bent elementary $3 \times 3$ square $\{(0, j, 0),(0, j, 1),(1, j, 1), j=0,1,2\}$ in our cube. In this elementary square the values of the field $u$ are given at eight points and the value of the field $u$ at the remaining ninth point $(1,2,1)$ is determined by relation (4), i.e., by the condition that the determinant of the matrix of values of the field at the lattice points of this bent elementary $3 \times 3$ square vanishes, because the corresponding $2 \times 2$ minor formed by initial data is not equal to zero. Since basic vectors of the same type are linearly independent, the vector of values of the field $u$ at the points of the line $\{(1, j, 1), 0 \leq j \leq 2\}$ is a linear combination of the vectors of values of the field $u$ at the points of the two basic lines of the same type, $\{(0, j, 0), 0 \leq j \leq 2\}$ and $\{(0, j, 1), 0 \leq j \leq 2\}$, situated in the given bent elementary $3 \times 3$ square; i.e., we can mark the point $(1,2,1)$ and shade the line $\{(1, j, 1), 0 \leq j \leq 2\}$. Moreover, the obtained vector of values of the field $u$ at the points of the line $\{(1, j, 1), 0 \leq j \leq 2\}$ forms a linearly independent pair of vectors with each of the basic vectors of the same type, since otherwise the corresponding $2 \times 2$ minors formed by initial data must be equal to zero.

Note that the vectors of values of the field $u$ at the points of the shaded lines $\{(1, j, 0), 0 \leq j \leq$ $2\}$ and $\{(1, j, 1), 0 \leq j \leq 2\},\{(i, 1,0), 0 \leq i \leq 2\}$ and $\{(i, 1,1), 0 \leq i \leq 2\},\{(0,1, k), 0 \leq k \leq 2\}$ 
and $\{(1,1, k), 0 \leq k \leq 2\},\{(2,0, k), 0 \leq k \leq 2\}$ and $\{(1,1, k), 0 \leq k \leq 2\}$, are linearly independent, since otherwise the corresponding $2 \times 2$ minors formed by initial data must be equal to zero.

Let us consider one more elementary $3 \times 3$ square $\{(0, j, 0),(0, j, 1),(0, j, 2), j=0,1,2\}$ in our cube (a face of the cube). In this elementary square at the present moment the values of the field $u$ are already determined at eight points and the value of the field $u$ at the remaining ninth point $(0,2,2)$ is determined by relation $(4)$, i.e., by the condition that the determinant of the matrix of values of the field at the lattice points of this elementary $3 \times 3$ square vanishes, because the corresponding $2 \times 2$ minor formed by initial data is not equal to zero. Since basic vectors of the same type are linearly independent, the vector of values of the field $u$ at the points of the line $\{(0, j, 2), 0 \leq j \leq 2\}$ is a linear combination of the vectors of values of the field $u$ at the points of the two basic lines of the same type, $\{(0, j, 0), 0 \leq j \leq 2\}$ and $\{(0, j, 1), 0 \leq j \leq 2\}$, situated in the given elementary $3 \times 3$ square; i.e., we can mark the point $(0,2,2)$ and shade the line $\{(0, j, 2), 0 \leq j \leq 2\}$. But in this case we do not state that the obtained vector of values of the field $u$ at the points of the line $\{(0, j, 2), 0 \leq j \leq 2\}$ forms linearly independent pairs of vectors with basic vectors of the same type.

We note that if the vector of values of the field $u$ at the lattice points of an arbitrary line is a linear combination of the vectors of values of the field $u$ at the lattice points of two shaded lines of the same type, then this vector is a linear combination of the vectors of values of the field $u$ at the points of the two basic lines of the same type. This follows immediately from the fact that each vector of values of the field $u$ at the points of an arbitrary shaded line is a linear combination of the vectors of values of the field $u$ at the points of the two basic lines of the same type.

Since the determinant of the matrix of values of the field at the points of the elementary $3 \times 3$ square $\{(0, j, 0),(0, j, 1),(0, j, 2), j=0,1,2\}$ (on a face of our cube) vanishes and, as it was noted above, the vectors of values of the field $u$ at the points of the two shaded lines, $\{(0,0, k), 0 \leq k \leq 2\}$ and $\{(0,1, k), 0 \leq k \leq 2\}$, are linearly independent, it follows immediately that the vector of values of the field $u$ at the points of the line $\{(0,2, k), 0 \leq k \leq 2\}$ is a linear combination of the vectors of values of the field $u$ at the points of these two lines of this elementary $3 \times 3$ square, namely, two shaded lines of the same type, $\{(0,0, k), 0 \leq k \leq 2\}$ and $\{(0,1, k), 0 \leq k \leq 2\}$, situated in the given elementary $3 \times 3$ square; i.e., we can shade the line $\{(0,2, k), 0 \leq k \leq 2\}$. Moreover, the obtained vector of values of the field $u$ at the points of the line $\{(0,2, k), 0 \leq k \leq 2\}$ forms a linearly independent pair of vectors with the vector of values of the field $u$ at the points of the basic line $\{(0,0, k), 0 \leq k \leq 2\}$ and also with the vector of values of the field $u$ at the points of the line $\{(0,1, k), 0 \leq k \leq 2\}$ and the vector of values of the field $u$ at the points of the line $\{(1,1, k), 0 \leq k \leq 2\}$, since otherwise the corresponding $2 \times 2$ minors formed by initial data must be equal to zero.

In the elementary $3 \times 3$ square $\{(i, 0,0),(i, 1,0),(i, 2,0), i=0,1,2\}$ of our cube (on a face of the cube) at the present moment the values of the field $u$ are already determined at eight points and the value of the field $u$ at the remaining ninth point $(2,2,0)$ is determined by relation (4), i.e., by the condition that the determinant of the matrix of values of the field at the points of this elementary $3 \times 3$ square vanishes, because the corresponding $2 \times 2$ minor formed by initial data is not equal to zero. Since, as it was noted above, the vectors of values of the field $u$ at the points of the two shaded lines, $\{(i, 0,0), 0 \leq i \leq 2\}$ and $\{(i, 1,0), 0 \leq i \leq 2\}$, are linearly independent, the vector of values of the field $u$ at the points of the line $\{(i, 2,0), 0 \leq i \leq 2\}$ is a linear combination of the vectors of values of the field $u$ at the points of these two shaded lines of the same type, $\{(i, 0,0), 0 \leq i \leq 2\}$ and $\{(i, 1,0), 0 \leq i \leq 2\}$, situated in the given elementary $3 \times 3$ square; i.e., we can mark the point $(2,2,0)$ and shade the line $\{(i, 2,0), 0 \leq i \leq 2\}$. But in this case we do not state that the obtained vector of values of the field $u$ at the points of the line $\{(i, 2,0), 0 \leq i \leq 2\}$ forms linearly independent pairs of vectors with other vectors of values of the field $u$ at the points of shaded lines of the same type. 
Since the determinant of the matrix of values of the field at the points of the elementary $3 \times 3$ square $\{(i, 0,0),(i, 1,0),(i, 2,0), i=0,1,2\}$ (on a face of our cube) vanishes and, as it was noted above, the vectors of values of the field $u$ at the points of the two shaded lines, $\{(0, j, 0), 0 \leq j \leq 2\}$ and $\{(1, j, 0), 0 \leq j \leq 2\}$, are linearly independent, it follows immediately that the vector of values of the field $u$ at the points of the line $\{(2, j, 0), 0 \leq j \leq 2\}$ is a linear combination of the vectors of values of the field $u$ at the points of two other lines of this elementary $3 \times 3$ square, namely, two shaded lines of the same type, $\{(0, j, 0), 0 \leq j \leq 2\}$ and $\{(1, j, 0), 0 \leq j \leq 2\}$, situated in the given elementary $3 \times 3$ square; i.e., we can shade the line $\{(2, j, 0), 0 \leq j \leq 2\}$. But in this case we do not state that the obtained vector of values of the field $u$ at the points of the line $\{(2, j, 0), 0 \leq j \leq 2\}$ forms linearly independent pairs of vectors with other vectors of values of the field $u$ at the points of shaded lines of the same type.

In the elementary $3 \times 3$ square $\{(i, 0,1),(i, 1,1),(i, 2,1), i=0,1,2\}$ of our cube (on a middle normal section of the cube) at the present moment the values of the field $u$ are already determined at eight points and the value of the field $u$ at the remaining ninth point $(2,2,1)$ is determined by relation (4), i.e., by the condition that the determinant of the matrix of values of the field at the points of this elementary $3 \times 3$ square vanishes, because the corresponding $2 \times 2$ minor formed by initial data is not equal to zero. Since, as it was noted above, the vectors of values of the field $u$ at the points of the two shaded lines, $\{(i, 0,1), 0 \leq i \leq 2\}$ and $\{(i, 1,1), 0 \leq i \leq 2\}$, are linearly independent, the vector of values of the field $u$ at the points of the line $\{(i, 2,1), 0 \leq i \leq 2\}$ is a linear combination of the vectors of values of the field $u$ at the points of these two shaded lines of the same type, $\{(i, 0,1), 0 \leq i \leq 2\}$ and $\{(i, 1,1), 0 \leq i \leq 2\}$, situated in the given elementary $3 \times 3$ square; i.e., we can mark the point $(2,2,1)$ and shade the line $\{(i, 2,1), 0 \leq i \leq 2\}$.

Since the determinant of the matrix of values of the field at the points of the elementary $3 \times 3$ square $\{(i, 0,1),(i, 1,1),(i, 2,1), i=0,1,2\}$ (on a middle normal section of our cube) vanishes and, as it was noted above, the vectors of values of the field $u$ at the points of the two shaded lines, $\{(0, j, 1), 0 \leq j \leq 2\}$ and $\{(1, j, 1), 0 \leq j \leq 2\}$, are linearly independent, it follows immediately that the vector of values of the field $u$ at the points of the line $\{(2, j, 1), 0 \leq j \leq 2\}$ is a linear combination of the vectors of values of the field $u$ at the points of two other lines of this elementary $3 \times 3$ square, namely, two shaded lines of the same type, $\{(0, j, 1), 0 \leq j \leq 2\}$ and $\{(1, j, 1), 0 \leq j \leq 2\}$, situated in the given elementary $3 \times 3$ square; i.e., we can shade the line $\{(2, j, 1), 0 \leq j \leq 2\}$.

Let us consider one more elementary $3 \times 3$ square $\{(1, j, 0),(1, j, 1),(1, j, 2), j=0,1,2\}$ in our cube (a middle normal section of the cube). In this elementary square at the present moment the values of the field $u$ are already determined at eight points and the value of the field $u$ at the remaining ninth point $(1,2,2)$ is determined by relation (4), i.e., by the condition that the determinant of the matrix of values of the field at the points of this elementary $3 \times 3$ square vanishes, because the corresponding $2 \times 2$ minor formed by initial data is not equal to zero. Since, as it was noted above, the vectors of values of the field $u$ at the points of the two shaded lines, $\{(1, j, 0), 0 \leq j \leq 2\}$ and $\{(1, j, 1), 0 \leq j \leq 2\}$, are linearly independent, the vector of values of the field $u$ at the points of the line $\{(1, j, 2), 0 \leq j \leq 2\}$ is a linear combination of the vectors of values of the field $u$ at the points of these two shaded lines of the same type, $\{(1, j, 0), 0 \leq j \leq 2\}$ and $\{(1, j, 1), 0 \leq j \leq 2\}$, situated in the given elementary $3 \times 3$ square; i.e., we can mark the point $(1,2,2)$ and shade the line $\{(1, j, 2), 0 \leq j \leq 2\}$.

Since the determinant of the matrix of values of the field at the points of the elementary $3 \times 3$ square $\{(1, j, 0),(1, j, 1),(1, j, 2), j=0,1,2\}$ (on a middle normal section of our cube) vanishes and, as it was noted above, the vectors of values of the field $u$ at the points of the two shaded lines, $\{(1,0, k), 0 \leq k \leq 2\}$ and $\{(1,1, k), 0 \leq k \leq 2\}$, are linearly independent, it follows immediately that the vector of values of the field $u$ at the points of the line $\{(1,2, k), 0 \leq k \leq 2\}$ is a linear combination of the vectors of values of the field $u$ at the points of two other lines of this 
elementary $3 \times 3$ square, namely, two shaded lines of the same type, $\{(1,0, k), 0 \leq k \leq 2\}$ and $\{(1,1, k), 0 \leq k \leq 2\}$, situated in the given elementary $3 \times 3$ square; i.e., we can shade the line $\{(1,2, k), 0 \leq k \leq 2\}$.

Let us consider one more elementary $3 \times 3$ square $\{(i, 1,0),(i, 1,1),(i, 1,2), i=0,1,2\}$ in our cube (a middle normal section of the cube). In this elementary square at the present moment the values of the field $u$ are already determined at eight points and the value of the field $u$ at the remaining ninth point $(2,1,2)$ is determined by relation (4), i.e., by the condition that the determinant of the matrix of values of the field at the points of this elementary $3 \times 3$ square vanishes, because the corresponding $2 \times 2$ minor formed by initial data is not equal to zero. Since, as it was noted above, the vectors of values of the field $u$ at the points of the two shaded lines, $\{(i, 1,0), 0 \leq i \leq 2\}$ and $\{(i, 1,1), 0 \leq i \leq 2\}$, are linearly independent, the vector of values of the field $u$ at the points of the line $\{(i, 1,2), 0 \leq i \leq 2\}$ is a linear combination of the vectors of values of the field $u$ at the points of these two shaded lines of the same type, $\{(i, 1,0), 0 \leq i \leq 2\}$ and $\{(i, 1,1), 0 \leq i \leq 2\}$, situated in the given elementary $3 \times 3$ square; i.e., we can mark the point $(2,1,2)$ and shade the line $\{(i, 1,2), 0 \leq i \leq 2\}$.

Since the determinant of the matrix of values of the field at the points of the elementary $3 \times 3$ square $\{(i, 1,0),(i, 1,1),(i, 1,2), i=0,1,2\}$ (on a middle normal section of our cube) vanishes and, as it was noted above, the vectors of values of the field $u$ at the points of the two shaded lines, $\{(0,1, k), 0 \leq k \leq 2\}$ and $\{(1,1, k), 0 \leq k \leq 2\}$, are linearly independent, it follows immediately that the vector of values of the field $u$ at the points of the line $\{(2,1, k), 0 \leq k \leq 2\}$ is a linear combination of the vectors of values of the field $u$ at the points of two other lines of this elementary $3 \times 3$ square, namely, two shaded lines of the same type, $\{(0,1, k), 0 \leq k \leq 2\}$ and $\{(1,1, k), 0 \leq k \leq 2\}$, situated in the given elementary $3 \times 3$ square; i.e., we can shade the line $\{(2,1, k), 0 \leq k \leq 2\}$.

It remains to determine the value of the field $u$ only at one point $(2,2,2)$ of our cube, and only three edges of the cube that contain this point are still unshaded for the present.

In order to determine the value of the field $u$ at the remaining lattice point $(2,2,2)$, it is necessary to show that in our cube there is at least one elementary $3 \times 3$ square (bent or unbent) containing this point $(2,2,2)$ and such that the corresponding $2 \times 2$ minor in the $3 \times 3$ matrix of values of the field $u$ at the points of this elementary $3 \times 3$ square is not equal to zero, since otherwise any value of the field $u$ at the point $(2,2,2)$ could not be determined. Note that in our cube the lattice point $(2,2,2)$ is the only one for which in each elementary $3 \times 3$ square (bent or unbent) containing this lattice point the corresponding $2 \times 2$ minor is not completely formed by initial data.

Let us prove that the determinant of the $2 \times 2$ matrix of values of the field $u$ at the points $\{(0,1,1),(1,1,1),(0,2,1),(1,2,1)\}$ of our cube is not equal to zero. Let us assume that it vanishes. In this case, the 2 -vectors $\left(u_{011}, u_{021}\right)$ and $\left(u_{111}, u_{121}\right)$ must be linearly dependent, and since the vector $\left(u_{011}, u_{021}\right)$ of initial data must not be zero (otherwise the corresponding $2 \times 2$ minor formed by initial data must vanish), the vector $\left(u_{111}, u_{121}\right)$ is proportional to the vector $\left(u_{011}, u_{021}\right)$ :

$$
\left(u_{111}, u_{121}\right)=\lambda\left(u_{011}, u_{021}\right) .
$$

On the other hand, as it was noted above, the vector $\left(u_{101}, u_{111}, u_{121}\right)$ of values of the field $u$ is a linear combination of the basic vectors of the same type $\left(u_{000}, u_{010}, u_{020}\right)$ and $\left(u_{001}, u_{011}, u_{021}\right)$ :

$$
\left(u_{101}, u_{111}, u_{121}\right)=\alpha\left(u_{000}, u_{010}, u_{020}\right)+\beta\left(u_{001}, u_{011}, u_{021}\right),
$$

and in this case

$$
\left(u_{111}, u_{121}\right)=\alpha\left(u_{010}, u_{020}\right)+\beta\left(u_{011}, u_{021}\right) .
$$


Using relation (5) we obtain

$$
\lambda\left(u_{011}, u_{021}\right)=\alpha\left(u_{010}, u_{020}\right)+\beta\left(u_{011}, u_{021}\right) .
$$

Hence, since the 2 -vectors $\left(u_{011}, u_{021}\right)$ and $\left(u_{010}, u_{020}\right)$ are linearly independent (otherwise the corresponding $2 \times 2$ minor formed by initial data must vanish), we have $\alpha=0$, i.e., relation (6) assumes the form

$$
\left(u_{101}, u_{111}, u_{121}\right)=\beta\left(u_{001}, u_{011}, u_{021}\right)
$$

but this is impossible, because in this case the corresponding $2 \times 2$ minor formed by initial data must vanish.

Thus, it is proved that the determinant of the $2 \times 2$ matrix of values of the field $u$ at the points $\{(0,1,1),(1,1,1),(0,2,1),(1,2,1)\}$ of our cube is not equal to zero.

Let us consider the bent elementary $3 \times 3$ square $\{(i, 1,1),(i, 2,1),(i, 2,2), i=0,1,2\}$ in our cube. In this elementary square at the present moment the values of the field $u$ are already determined at eight points and the value of the field $u$ at the remaining ninth point $(2,2,2)$ is determined by relation (4), i.e., by the condition that the determinant of the matrix of values of the field at the lattice points of this elementary $3 \times 3$ square vanishes, because we proved that the corresponding $2 \times 2$ minor is not equal to zero. Since the vectors of values of the field $u$ at the points of the two shaded lines, $\{(i, 2,1), 0 \leq i \leq 2\}$ and $\{(i, 1,1), 0 \leq i \leq 2\}$, are linearly independent (otherwise the nonzero $2 \times 2$ minor considered above must vanish), the vector of values of the field $u$ at the points of the line $\{(i, 2,2), 0 \leq i \leq 2\}$ is a linear combination of the vectors of values of the field $u$ at the points of these two shaded lines of the same type, $\{(i, 2,1), 0 \leq i \leq 2\}$ and $\{(i, 1,1), 0 \leq i \leq 2\}$, situated in the given bent elementary $3 \times 3$ square; i.e., we can mark the point $(2,2,2)$ and shade the line $\{(i, 2,2), 0 \leq$ $i \leq 2\}$.

Now the values of the field $u$ are determined already at all points of our cube, and it remains to shade two edges of the cube.

At first we prove that the determinant of the $2 \times 2$ matrix of values of the field $u$ at the points $\{(0,1,1),(1,1,1),(0,1,2),(1,1,2)\}$ of our cube is not equal to zero. Let us assume that it vanishes. In this case, the 2 -vectors $\left(u_{011}, u_{012}\right)$ and $\left(u_{111}, u_{112}\right)$ must be linearly dependent. Moreover, both these vectors are nonzero, since otherwise the corresponding $2 \times 2$ minor formed by initial data must vanish. Indeed, let us assume, for example, that the 2vector $\left(u_{011}, u_{012}\right)$ is zero, i.e., $u_{011}=0, u_{012}=0$. Then, in the bent elementary $3 \times 3$ square $\{(0,1, k),(0,0, k),(1,0, k), k=0,1,2\}$ in our cube, the determinant of the matrix of values of the field $u$ at the points of this bent elementary $3 \times 3$ square is equal, except for sign, to the product of the value $u_{010}$ of the field $u$ by the corresponding $2 \times 2$ minor formed by initial data, and this $2 \times 2$ minor is not equal to zero by our condition on initial data. Thus, as the determinant of the matrix of values of the field at the points of this bent elementary $3 \times 3$ square vanishes, it follows that $u_{010}=0$, and since $u_{011}=0$, the corresponding $2 \times 2$ minor formed by initial data is equal to zero, but this is impossible. Similarly, it can be proved that the 2-vector $\left(u_{111}, u_{112}\right)$ is also nonzero. Indeed, let us assume that the 2vector $\left(u_{111}, u_{112}\right)$ is zero, i.e., $u_{111}=0, u_{112}=0$. Then, in the bent elementary $3 \times 3$ square $\{(1,1, k),(1,0, k),(0,0, k), k=0,1,2\}$ in our cube, the determinant of the matrix of values of the field $u$ at the points of this bent elementary $3 \times 3$ square is equal, except for sign, to the product of the value $u_{110}$ of the field $u$ by the corresponding $2 \times 2$ minor formed by initial data, and this $2 \times 2$ minor is not equal to zero by our condition on initial data. Thus, as the determinant of the matrix of values of the field at the points of this bent elementary $3 \times 3$ square vanishes, it follows that $u_{110}=0$, and since $u_{111}=0$, the corresponding $2 \times 2$ minor formed by initial data is equal to zero, but this is impossible. So we have proved that the 
2 -vectors $\left(u_{011}, u_{012}\right)$ and $\left(u_{111}, u_{112}\right)$ are nonzero. Moreover, these 2-vectors must be linearly dependent under our assumption. Therefore, each of these 2-vectors is proportional to the other:

$$
\left(u_{011}, u_{012}\right)=\lambda\left(u_{111}, u_{112}\right), \quad\left(u_{111}, u_{112}\right)=\mu\left(u_{011}, u_{012}\right) .
$$

On the other hand, since the determinant of the matrix of values of the field at the points of the bent elementary $3 \times 3$ square $\{(1,0, k),(1,1, k),(0,1, k), k=0,1,2\}$ vanishes and, as it was noted above, the vectors of values of the field $u$ at the points of the two shaded lines, $\{(1,1, k), 0 \leq k \leq 2\}$ and $\{(1,0, k), 0 \leq k \leq 2\}$, are linearly independent, it follows immediately that the vector of values of the field $u$ at the points of the line $\{(0,1, k), 0 \leq$ $k \leq 2\}$ is a linear combination of the vectors of values of the field $u$ at the points of two other lines of this bent elementary $3 \times 3$ square, namely, two shaded lines of the same type, $\{(1,1, k), 0 \leq k \leq 2\}$ and $\{(1,0, k), 0 \leq k \leq 2\}$, situated in the given bent elementary $3 \times 3$ square:

$$
\left(u_{010}, u_{011}, u_{012}\right)=\alpha\left(u_{110}, u_{111}, u_{112}\right)+\beta\left(u_{100}, u_{101}, u_{102}\right),
$$

and in this case

$$
\left(u_{011}, u_{012}\right)=\alpha\left(u_{111}, u_{112}\right)+\beta\left(u_{101}, u_{102}\right) .
$$

Using relation (7), we obtain

$$
\lambda\left(u_{111}, u_{112}\right)=\alpha\left(u_{111}, u_{112}\right)+\beta\left(u_{101}, u_{102}\right) .
$$

If the 2 -vectors $\left(u_{111}, u_{112}\right)$ and $\left(u_{101}, u_{102}\right)$ are linearly independent, i.e., the determinant of the matrix of values of the field $u$ at the points $\{(1,1,1),(1,0,1),(1,0,2),(1,1,2)\}$ of our cube is not equal to zero, then $\beta=0$ and relation (8) assumes the form

$$
\left(u_{010}, u_{011}, u_{012}\right)=\alpha\left(u_{110}, u_{111}, u_{112}\right)
$$

but this is impossible, since in this case the corresponding $2 \times 2$ minor formed by initial data must be equal to zero. Hence, under our assumptions the 2-vectors $\left(u_{111}, u_{112}\right)$ and $\left(u_{101}, u_{102}\right)$ must be linearly dependent, i.e., the determinant of $2 \times 2$ matrix of values of the field $u$ at the points $\{(1,1,1),(1,0,1),(1,0,2),(1,1,2)\}$ of our cube vanishes. Since the 2 -vector $\left(u_{111}, u_{112}\right)$ is nonzero and the determinant of the $2 \times 2$ matrix of values of the field $u$ at the points $\{(1,1,1),(1,0,1),(1,0,2),(1,1,2)\}$ of our cube vanishes, it follows that the 2 -vector $\left(u_{101}, u_{102}\right)$ is proportional to the 2 -vector $\left(u_{111}, u_{112}\right)$ :

$$
\left(u_{101}, u_{102}\right)=\nu\left(u_{111}, u_{112}\right) .
$$

Since the determinant of the matrix of values of the field at the points of the bent elementary $3 \times 3$ square $\{(1,1, k),(0,1, k),(0,0, k), k=0,1,2\}$ vanishes and, as it was noted above, the vectors of values of the field $u$ at the points of the two shaded lines, $\{(0,1, k), 0 \leq k \leq 2\}$ and $\{(0,0, k), 0 \leq k \leq 2\}$, are linearly independent, it follows immediately that the vector of values of the field $u$ at the points of the line $\{(1,1, k), 0 \leq k \leq 2\}$ is a linear combination of the vectors of values of the field $u$ at the points of two other lines of this bent elementary $3 \times 3$ square, namely, two shaded lines of the same type, $\{(0,1, k), 0 \leq k \leq 2\}$ and $\{(0,0, k), 0 \leq k \leq 2\}$, situated in the given bent elementary $3 \times 3$ square:

$$
\left(u_{110}, u_{111}, u_{112}\right)=\alpha\left(u_{010}, u_{011}, u_{012}\right)+\beta\left(u_{000}, u_{001}, u_{002}\right),
$$


and in this case

$$
\left(u_{111}, u_{112}\right)=\alpha\left(u_{011}, u_{012}\right)+\beta\left(u_{001}, u_{002}\right) .
$$

Using relation (7), we obtain

$$
\mu\left(u_{011}, u_{012}\right)=\alpha\left(u_{011}, u_{012}\right)+\beta\left(u_{001}, u_{002}\right) .
$$

If the 2-vectors $\left(u_{011}, u_{012}\right)$ and $\left(u_{001}, u_{002}\right)$ are linearly independent, i.e., the determinant of the matrix of values of the field $u$ at the points $\{(0,1,1),(0,0,1),(0,0,2),(0,1,2)\}$ of our cube is not equal to zero, then $\beta=0$ and relation (10) assumes the form

$$
\left(u_{110}, u_{111}, u_{112}\right)=\alpha\left(u_{010}, u_{011}, u_{012}\right),
$$

but this is impossible, since in this case the corresponding $2 \times 2$ minor formed by initial data must be equal to zero. Hence, under our assumptions the 2 -vectors $\left(u_{011}, u_{012}\right)$ and $\left(u_{001}, u_{002}\right)$ must be linearly dependent, i.e., the determinant of the $2 \times 2$ matrix of values of the field $u$ at the points $\{(0,1,1),(0,0,1),(0,0,2),(0,1,2)\}$ of our cube vanishes. Since the 2 -vector $\left(u_{011}, u_{012}\right)$ is nonzero and the determinant of the $2 \times 2$ matrix of values of the field $u$ at the points $\{(0,1,1),(0,0,1),(0,0,2),(0,1,2)\}$ of our cube vanishes, it follows that the 2 -vector $\left(u_{001}, u_{002}\right)$ is proportional to the 2 -vector $\left(u_{011}, u_{012}\right)$ :

$$
\left(u_{001}, u_{002}\right)=\varkappa\left(u_{011}, u_{012}\right) .
$$

Using relation (7), we obtain

$$
\left(u_{001}, u_{002}\right)=\varkappa\left(u_{011}, u_{012}\right)=\varkappa \lambda\left(u_{111}, u_{112}\right)
$$

and from (9) we have

$$
\left(u_{101}, u_{102}\right)=\nu\left(u_{111}, u_{112}\right),
$$

i.e., the 2-vectors $\left(u_{001}, u_{002}\right)$ and $\left(u_{101}, u_{102}\right)$ are linearly dependent, but this is impossible, since in this case the corresponding $2 \times 2$ minor formed by initial data must be equal to zero.

Thus, it is proved that the determinant of the $2 \times 2$ matrix of values of the field $u$ at the points $\{(0,1,1),(1,1,1),(0,1,2),(1,1,2)\}$ of our cube is not equal to zero.

Since the determinant of the matrix of values of the field $u$ at the points of the bent elementary $3 \times 3$ square $\{(i, 0,2),(i, 1,2),(i, 1,1), i=0,1,2\}$ vanishes (the three lines $\{(i, 0,2), i=0,1,2\}$, $\{(i, 1,2), i=0,1,2\}$ and $\{(i, 1,1), i=0,1,2\}$ are shaded in this bent elementary $3 \times 3$ square), it follows immediately that the three vectors of values of the field $u$ at the points of the bent lines $\{(0,0,2),(0,1,2),(0,1,1)\},\{(1,0,2),(1,1,2),(1,1,1)\}$ and $\{(2,0,2),(2,1,2),(2,1,1)\}$ are linearly dependent; moreover, the vectors of values of the field $u$ at the points of the bent lines $\{(0,0,2),(0,1,2),(0,1,1)\}$ and $\{(1,0,2),(1,1,2),(1,1,1)\}$ are linearly independent, since otherwise the nonzero determinant of the $2 \times 2$ matrix of values of the field $u$ at the points $\{(0,1,1),(1,1,1),(0,1,2),(1,1,2)\}$ of our cube must vanish. Thus, the vector of values of the field $u$ at the points of the bent line $\{(2,0,2),(2,1,2),(2,1,1)\}$ is a linear combination of the vectors of values of the field $u$ at the points of the bent lines $\{(0,0,2),(0,1,2),(0,1,1)\}$ and $\{(1,0,2),(1,1,2),(1,1,1)\}$ :

$$
\left(u_{202}, u_{212}, u_{211}\right)=\alpha\left(u_{002}, u_{012}, u_{011}\right)+\beta\left(u_{102}, u_{112}, u_{111}\right) .
$$

Similarly, since the determinant of the matrix of values of the field at the points of the bent elementary $3 \times 3$ square $\{(i, 2,2),(i, 1,2),(i, 1,1), i=0,1,2\}$ vanishes (the three lines $\{(i, 2,2), i=$ 
$0,1,2\},\{(i, 1,2), i=0,1,2\}$ and $\{(i, 1,1), i=0,1,2\}$ are shaded in this bent elementary $3 \times 3$ square), it follows immediately that the vectors of values of the field $u$ at the points of the bent lines $\{(0,2,2),(0,1,2),(0,1,1)\},\{(1,2,2),(1,1,2),(1,1,1)\}$ and $\{(2,2,2),(2,1,2),(2,1,1)\}$ are linearly dependent; moreover, the vectors of values of the field $u$ at the points of the bent lines $\{(0,2,2),(0,1,2),(0,1,1)\}$ and $\{(1,2,2),(1,1,2),(1,1,1)\}$ are linearly independent, since otherwise the nonzero determinant of the $2 \times 2$ matrix of values of the field $u$ at the points $\{(0,1,1),(1,1,1),(0,1,2),(1,1,2)\}$ of our cube must vanish. Thus, in the bent elementary $3 \times 3$ square under consideration, the vector of values of the field $u$ at the points of the bent line $\{(2,2,2),(2,1,2),(2,1,1)\}$ is a linear combination of the vectors of values of the field $u$ at the points of the bent lines $\{(0,2,2),(0,1,2),(0,1,1)\}$ and $\{(1,2,2),(1,1,2),(1,1,1)\}$ :

$$
\left(u_{222}, u_{212}, u_{211}\right)=\gamma\left(u_{022}, u_{012}, u_{011}\right)+\delta\left(u_{122}, u_{112}, u_{111}\right) .
$$

From relations (11) and (12), we obtain respectively

$$
\left(u_{212}, u_{211}\right)=\alpha\left(u_{012}, u_{011}\right)+\beta\left(u_{112}, u_{111}\right)
$$

and

$$
\left(u_{212}, u_{211}\right)=\gamma\left(u_{012}, u_{011}\right)+\delta\left(u_{112}, u_{111}\right),
$$

whence it follows immediately that $\alpha=\gamma$ and $\beta=\delta$, since the determinant of the $2 \times 2$ matrix of values of the field $u$ at the points $\{(0,1,1),(1,1,1),(0,1,2),(1,1,2)\}$ of our cube is not equal to zero and the 2 -vectors $\left(u_{011}, u_{012}\right)$ and $\left(u_{111}, u_{112}\right)$ are linearly independent. From relations $(11)$ and (12), we obtain respectively

$$
\left(u_{202}, u_{212}\right)=\alpha\left(u_{002}, u_{012}\right)+\beta\left(u_{102}, u_{112}\right)
$$

and

$$
\left(u_{222}, u_{212}\right)=\gamma\left(u_{022}, u_{012}\right)+\delta\left(u_{122}, u_{112}\right) .
$$

Since $\alpha=\gamma$ and $\beta=\delta$, from relations (13) and (14)

$$
\left(u_{202}, u_{212}, u_{222}\right)=\alpha\left(u_{002}, u_{012}, u_{022}\right)+\beta\left(u_{102}, u_{112}, u_{122}\right),
$$

i.e., the vector of values of the field $u$ at the points of the line $\{(2,2,2),(2,1,2),(2,0,2)\}$ is a linear combination of the vectors of values of the field $u$ at the points of the two shaded lines $\{(0,2,2),(0,1,2),(0,0,2)\}$ and $\{(1,2,2),(1,1,2),(1,0,2)\}$, and hence we can shade also the line $\{(2,2,2),(2,1,2),(2,0,2)\}$ in our cube.

Let us prove now that the determinant of the $2 \times 2$ matrix of values of the field $u$ at the points $\{(1,0,1),(1,1,1),(2,1,1),(2,0,1)\}$ of our cube is not equal to zero. Let us assume that it vanishes. In this case, the 2 -vectors $\left(u_{111}, u_{211}\right)$ and $\left(u_{101}, u_{201}\right)$ must be linearly dependent. Moreover, the vector $\left(u_{101}, u_{201}\right)$ is nonzero, since otherwise the corresponding $2 \times 2$ minor formed by initial data must vanish. In this case the 2 -vector $\left(u_{111}, u_{211}\right)$ must be proportional to the 2 -vector $\left(u_{101}, u_{201}\right)$ :

$$
\left(u_{111}, u_{211}\right)=\lambda\left(u_{101}, u_{201}\right) .
$$

On the other hand, since the determinant of the matrix of values of the field at the points of the bent elementary $3 \times 3$ square $\{(i, 0,0),(i, 0,1),(i, 1,1), i=0,1,2\}$ vanishes and the vectors of values of the field $u$ at the points of the two basic lines $\{(i, 0,0), 0 \leq i \leq 2\}$ and $\{(i, 0,1), 0 \leq i \leq 2\}$ are linearly independent, it follows immediately that the vector of values of the field $u$ at the points of the line $\{(i, 1,1), 0 \leq i \leq 2\}$ is a linear combination of the vectors of 
values of the field $u$ at the points of the two lines of this bent elementary $3 \times 3$ square, namely, the two basic lines of the same type $\{(i, 0,0), 0 \leq i \leq 2\}$ and $\{(i, 0,1), 0 \leq i \leq 2\}$, situated in the given bent elementary $3 \times 3$ square:

$$
\left(u_{011}, u_{111}, u_{211}\right)=\alpha\left(u_{001}, u_{101}, u_{201}\right)+\beta\left(u_{000}, u_{100}, u_{200}\right),
$$

and in this case

$$
\left(u_{111}, u_{211}\right)=\alpha\left(u_{101}, u_{201}\right)+\beta\left(u_{100}, u_{200}\right) .
$$

Using relation (15), we obtain

$$
\lambda\left(u_{101}, u_{201}\right)=\alpha\left(u_{101}, u_{201}\right)+\beta\left(u_{100}, u_{200}\right) .
$$

Since the 2 -vectors $\left(u_{101}, u_{201}\right)$ and $\left(u_{100}, u_{200}\right)$ are linearly independent (otherwise the corresponding $2 \times 2$ minor formed by initial data must vanish), we have $\beta=0$ and relation (16) assumes the form

$$
\left(u_{011}, u_{111}, u_{211}\right)=\alpha\left(u_{001}, u_{101}, u_{201}\right),
$$

but this is impossible, because in this case the corresponding $2 \times 2$ minor formed by initial data must vanish.

Thus, it is proved that the determinant of the $2 \times 2$ matrix of values of the field $u$ at the points $\{(1,0,1),(1,1,1),(2,1,1),(2,0,1)\}$ of our cube is not equal to zero.

Since the determinant of the matrix of values of the field at the points of the bent elementary $3 \times 3$ square $\{(2, j, 0),(2, j, 1),(1, j, 1), j=0,1,2\}$ vanishes (the three lines $\{(2, j, 0), j=0,1,2\}$, $\{(2, j, 1), j=0,1,2\}$ and $\{(1, j, 1), j=0,1,2\}$ are shaded in this bent elementary $3 \times 3$ square), it follows immediately that the vectors of values of the field $u$ at the points of the bent lines $\{(2,0,0),(2,0,1),(1,0,1)\},\{(2,1,0),(2,1,1),(1,1,1)\}$ and $\{(2,2,0),(2,2,1),(1,2,1)\}$ are linearly dependent; moreover, the vectors of values of the field $u$ at the points of the bent lines $\{(2,0,0),(2,0,1),(1,0,1)\}$ and $\{(2,1,0),(2,1,1),(1,1,1)\}$ are linearly independent, since otherwise the nonzero determinant of the $2 \times 2$ matrix of values of the field $u$ at the points $\{(1,0,1),(1,1,1),(2,1,1),(2,0,1)\}$ of our cube must vanish. Thus, the vector of values of the field $u$ at the points of the bent line $\{(2,2,0),(2,2,1),(1,2,1)\}$ is a linear combination of the vectors of values of the field $u$ at the points of the bent lines $\{(2,0,0),(2,0,1),(1,0,1)\}$ and $\{(2,1,0),(2,1,1),(1,1,1)\}$ :

$$
\left(u_{220}, u_{221}, u_{121}\right)=\alpha\left(u_{200}, u_{201}, u_{101}\right)+\beta\left(u_{210}, u_{211}, u_{111}\right) .
$$

Similarly, since the determinant of the matrix of values of the field at the points of the bent elementary $3 \times 3$ square $\{(2, j, 2),(2, j, 1),(1, j, 1), j=0,1,2\}$ vanishes (the three lines $\{(2, j, 2), j=$ $0,1,2\},\{(2, j, 1), j=0,1,2\}$ and $\{(1, j, 1), j=0,1,2\}$ are shaded in this bent elementary $3 \times 3$ square), it follows immediately that the vectors of values of the field $u$ at the points of the bent lines $\{(1,0,1),(2,0,1),(2,0,2)\},\{(1,1,1),(2,1,1),(2,1,2)\}$ and $\{(1,2,1),(2,2,1),(2,2,2)\}$ are linearly dependent; moreover, the vectors of values of the field $u$ at the points of the bent lines $\{(1,0,1),(2,0,1),(2,0,2)\}$ and $\{(1,1,1),(2,1,1),(2,1,2)\}$ are linearly independent, since otherwise the nonzero determinant of the $2 \times 2$ matrix of values of the field $u$ at the points $\{(1,0,1),(1,1,1),(2,1,1),(2,0,1)\}$ of our cube must vanish. Thus, in the bent elementary $3 \times 3$ square under consideration, the vector of values of the field $u$ at the points of the bent line $\{(1,2,1),(2,2,1),(2,2,2)\}$ is a linear combination of the vectors of values of the field $u$ at the points of the bent lines $\{(1,0,1),(2,0,1),(2,0,2)\}$ and $\{(1,1,1),(2,1,1),(2,1,2)\}$ :

$$
\left(u_{222}, u_{221}, u_{121}\right)=\gamma\left(u_{202}, u_{201}, u_{101}\right)+\delta\left(u_{212}, u_{211}, u_{111}\right) .
$$


From relations (17) and (18), we obtain respectively

$$
\left(u_{221}, u_{121}\right)=\alpha\left(u_{201}, u_{101}\right)+\beta\left(u_{211}, u_{111}\right)
$$

and

$$
\left(u_{221}, u_{121}\right)=\gamma\left(u_{201}, u_{101}\right)+\delta\left(u_{211}, u_{111}\right),
$$

whence it follows immediately that $\alpha=\gamma$ and $\beta=\delta$, since the determinant of the $2 \times 2$ matrix of values of the field $u$ at the points $\{(1,0,1),(1,1,1),(2,1,1),(2,0,1)\}$ of our cube is not equal to zero and the 2 -vectors $\left(u_{201}, u_{101}\right)$ and $\left(u_{211}, u_{111}\right)$ are linearly independent. From relations $(17)$ and (18), we obtain respectively

$$
\left(u_{220}, u_{221}\right)=\alpha\left(u_{200}, u_{201}\right)+\beta\left(u_{210}, u_{211}\right)
$$

and

$$
\left(u_{222}, u_{221}\right)=\gamma\left(u_{202}, u_{201}\right)+\delta\left(u_{212}, u_{211}\right) .
$$

Since $\alpha=\gamma$ and $\beta=\delta$, from relations (19) and (20)

$$
\left(u_{222}, u_{221}, u_{220}\right)=\alpha\left(u_{202}, u_{201}, u_{200}\right)+\beta\left(u_{212}, u_{211}, u_{210}\right) \text {, }
$$

i.e., the vector of values of the field $u$ at the points of the line $\{(2,2,2),(2,2,1),(2,2,0)\}$ is a linear combination of the vectors of values of the field $u$ at the points of the two shaded lines $\{(2,0,2),(2,0,1),(2,0,0)\}$ and $\{(2,1,2),(2,1,1),(2,1,0)\}$, and hence we can shade also the line $\{(2,2,2),(2,2,1),(2,2,0)\}$ in our cube.

Thus, the values of the field $u$ are determined at all points of our cube, and all lines of the cube are shaded now. The theorem is proved.

Moreover, we have proved a considerably stronger principle of consistency on the cubic lattice for determinants.

Theorem 2 ([2]). For arbitrary generic initial data, the nonlinear discrete equation (4) can be satisfied in a consistent way and simultaneously on each set of points of three lines $P_{l}, 1 \leq l \leq 3$, of the cubic lattice $\mathbb{Z}^{3}$ of the form $P_{l}=\left\{\left(i, r_{l}, s_{l}\right), a \leq i \leq a+2\right\}, 1 \leq l \leq 3$, where $a, r_{l}$, and $s_{l}$, $1 \leq l \leq 3$, are arbitrary fixed integers ( $x$-type lines), as well as on each set of points of three lines $Q_{l}, 1 \leq l \leq 3$, of the cubic lattice $\mathbb{Z}^{3}$ of the form $Q_{l}=\left\{\left(r_{l}, j, s_{l}\right), a \leq j \leq a+2\right\}, 1 \leq l \leq 3$, where $a, r_{l}$, and $s_{l}, 1 \leq l \leq 3$, are arbitrary fixed integers (y-type lines), and on each set of points of three lines $R_{l}, 1 \leq l \leq 3$, of the cubic lattice $\mathbb{Z}^{3}$ of the form $R_{l}=\left\{\left(r_{l}, s_{l}, k\right), a \leq k \leq a+2\right\}$, $1 \leq l \leq 3$, where $a, r_{l}$, and $s_{l}, 1 \leq l \leq 3$, are arbitrary fixed integers ( $z$-type lines). Moreover, in this case the discrete equation (4) will be satisfied in a consistent way and simultaneously on each set of points of special form lying on three bent lines $S_{l}, 1 \leq l \leq 3$, of the same type in the cubic lattice $\mathbb{Z}^{3}$, for example, of the form $S_{l}=\left\{\left(a, r_{l}, s\right),\left(a+1, r_{l}, s\right),\left(a+1, r_{l}, s+1\right)\right\}$, $1 \leq l \leq 3$, where $a, r_{l}$, and $s, 1 \leq l \leq 3$, are arbitrary fixed integers, of the form $S_{l}=$ $\left\{\left(a, s, r_{l}\right),\left(a+1, s, r_{l}\right),\left(a+1, s+1, r_{l}\right)\right\}, 1 \leq l \leq 3$, where $a, r_{l}$, and $s, 1 \leq l \leq 3$, are arbitrary fixed integers, or of the form $S_{l}=\left\{\left(r_{l}, s, a+1\right),\left(r_{l}, s, a\right),\left(r_{l}, s+1, a\right)\right\}, 1 \leq l \leq 3$, where $a, r_{l}$, and $s, 1 \leq l \leq 3$, are arbitrary fixed integers.

The following principle of consistency on the cubic lattice for determinants also holds.

Let us consider an arbitrary line $P$ (bent or unbent) given by three arbitrary neighboring points in the cubic lattice $\mathbb{Z}^{3}$. We consider an arbitrary set of three lines of the cubic lattice $\mathbb{Z}^{3}$ that are obtained from the line $P$ by translations in the lattice by vectors parallel to the (onedimensional or two-dimensional) space orthogonal to the line $P$ (i.e., orthogonal to the plane or 
to the straight line of $P$ depending on whether the line $P$ is bent or unbent). Then, for arbitrary generic initial data, the nonlinear discrete equation (4) can be satisfied in a consistent way and simultaneously on each such set of three lines of the cubic lattice $\mathbb{Z}^{3}$.

Similar properties of consistency on cubic lattices hold for determinants of arbitrary order $N \geq 2($ see $[1,2])$.

\section{Acknowledgements}

The work was carried out under partial financial support from the Russian Foundation for Basic Research (project no. 09-01-00762) and from the programme "Leading Scientific Schools" (project no. NSh-5413.2010.1).

\section{References}

[1] Mokhov O.I., On consistency of determinants on cubic lattices, Uspekhi Mat. Nauk 63 (2008), no. 6, 169-170 (English transl.: Russian Math. Surveys 63 (2008), no. 6, 1146-1148), arXiv:0809.2032.

[2] Mokhov O.I., Consistency on cubic lattices for determinants of arbitrary orders, in Geometry, Topology and Mathematical Physics, Tr. Mat. Inst. Steklova 266 (2009), 202-217 (English transl.: Proc. Steklov Inst. Math. 266 (2009), 195-209), arXiv:0910.2044.

[3] Nijhoff F.W., Walker A.J., The discrete and continuous Painlevé VI hierarchy and the Garnier systems, Glasg. Math. J. 43A (2001), 109-123, nlin.SI/0001054.

[4] Nijhoff F.W., Lax pair for the Adler (lattice Krichever-Novikov) system, Phys. Lett. A 297 (2002), 49-58, nlin.SI/0110027.

[5] Bobenko A.I., Suris Yu.B., Integrable systems on quad-graphs, Int. Math. Res. Not. 2002 (2002), no. 11, 573-611, nlin.SI/0110004.

[6] Adler V.E., Bobenko A.I., Suris Yu.B., Classification of integrable equations on quad-graphs. The consistency approach, Comm. Math. Phys. 233 (2003), 513-543, nlin.SI/0202024.

[7] Bobenko A.I., Suris Yu.B., Discrete differential geometry. Integrable structure, Graduate Studies in Mathematics, Vol. 98, American Mathematical Society, Providence, RI, 2008, math.DG/0504358.

[8] Bobenko A.I., Suris Yu.B., On organizing principles of discrete differential geometry. Geometry of spheres, Uspekhi Mat. Nauk 62 (2007), no. 1, 3-50 (English transl.: Russian Math. Surveys 62 (2007), no. 1, 1-43), math.DG/0608291.

[9] Veselov A.P., Integrable maps, Uspekhi Mat. Nauk 46 (1991), no. 5, 3-45 (English transl.: Russian Math. Surveys 46 (1991), no. 5, 1-51).

[10] Adler V.E., Bobenko A.I., Suris Yu.B., Discrete nonlinear hyperbolic equations: classification of integrable cases, Funct. Anal. Appl. 43 (2009), 3-17, arXiv:0705.1663.

[11] Tsarev S.P., Wolf Th., Classification of three-dimensional integrable scalar discrete equations, Lett. Math. Phys 84 (2008), 31-39, arXiv:0706.2464.

[12] Hietarinta J., A new two-dimensional lattice model that is 'consistent around a cube', J. Phys. A: Math. Gen. 37 (2004), L67-L73, nlin.SI/0311034.

[13] Adler V.E., Veselov A.P., Cauchy problem for integrable discrete equations on quad-graphs, Acta Appl. Math. 84 (2004), 237-262, math-ph/0211054.

[14] Adler V.E., Suris Yu.B., Q4: integrable master equation related to an elliptic curve, Int. Math. Res. Not. 2004 (2004), no. 47, 2523-2553, nlin.SI/0309030. 